Research Article

\title{
Public Project Portfolio Optimization under a Participatory Paradigm
}

\author{
Eduardo Fernandez, ${ }^{1}$ and Rafael Olmedo ${ }^{2}$ \\ ${ }^{1}$ Faculty of Engineering, Autonomous University of Sinaloa, Angel Flores s/n Centro, 80000 Culiaćan, SIN, Mexico \\ ${ }^{2}$ Faculty of Mathematic and Physic Sciences, Autonomous University of Sinaloa, Angel Flores s/n Centro, 80000 Culiaćan, SIN, Mexico
}

Correspondence should be addressed to Rafael Olmedo; rolmedo@uas.uasnet.mx

Received 30 May 2012; Revised 4 October 2012; Accepted 12 October 2012

Academic Editor: Qingfu Zhang

Copyright (c) 2013 E. Fernandez and R. Olmedo. This is an open access article distributed under the Creative Commons Attribution License, which permits unrestricted use, distribution, and reproduction in any medium, provided the original work is properly cited.

\begin{abstract}
A new democracy paradigm is emerging through participatory budgeting exercises, which can be defined as a public space in which the government and the society agree on how to adapt the priorities of the citizenship to the public policy agenda. Although these priorities have been identified and they are likely to be reflected in a ranking of public policy actions, there is still a challenge of solving a portfolio problem of public projects that should implement the agreed agenda. This work proposes two procedures for optimizing the portfolio of public actions with the information stemming from the citizen participatory exercise. The selection of the method depends on the information about preferences collected from the participatory group. When the information is sufficient, the method behaves as an instrument of legitimate democracy. The proposal performs very well in solving two real-size examples.
\end{abstract}

\section{Introduction}

Even in the best scenarios, designing public policies is far from being an exact science, with quantitative determinations beyond all subjectivity. Without denying the objective content of the social interest, it is certain that the difficulty in apprehending it opens space to methods that seek to model the preferences of concrete individuals, able to express their preferences in a more or less consistent way.

Up to now, democracy in the distribution of public resources has been fundamentally expressed in

(i) the action of groups empowered by the society to make budget decisions on its behalf (GESBD) (parliaments, communes, governing boards of public organizations), formed by members of the political class legitimized by the popular vote, but that respond to their personal and their party's interests instead of to the will of the electorate,

(ii) the action of GESBDs formed by officials and experts appointed by the executive power that rather than interests of the electorate, reflected only in a very indirect way, reflect policies already designed by the executive.

(iii) the attempts of participatory budgeting carried out at local level where the population's priorities are directly heard by constituted authorities and are later reflected in the distribution of resources once the compatibility with the opinions of the political class has been achieved.

Since its emergence in Porto Alegre, Brazil, participatory budgeting has spread to hundreds of Latin American cities and dozens of cities in other continents. "Participatory budgeting" can be defined as a public space in which the government and the society agree how to adapt the priorities of the citizenship to the public policy agenda. The utility of these participatory exercises is that the government obtains information about the priorities of the participating social sectors and thus can perhaps identify programs that are of consensual benefit.

However, the challenge that is still not approached by "participatory budgeting" is how to translate priorities and policies stemming from the exercise into a system of concrete social action projects, each with well-defined resources 
TABLE 1: Information about projects.

\begin{tabular}{|c|c|}
\hline Position in ranking & Requirements (million dollars) \\
\hline 1 & 84.00 \\
\hline 2 & 124.50 \\
\hline 3 & 129.75 \\
\hline 4 & 147.75 \\
\hline 5 & 126.00 \\
\hline 6 & 137.25 \\
\hline 7 & 96.00 \\
\hline 8 & 84.75 \\
\hline 9 & 93.00 \\
\hline 10 & 121.50 \\
\hline 11 & 102.75 \\
\hline 12 & 141.75 \\
\hline 13 & 105.75 \\
\hline 14 & 98.25 \\
\hline 15 & 101.25 \\
\hline 16 & 83.25 \\
\hline 17 & 109.50 \\
\hline 18 & 107.25 \\
\hline 19 & 135.00 \\
\hline 20 & 97.50 \\
\hline 21 & 127.50 \\
\hline 22 & 114.00 \\
\hline 23 & 106.50 \\
\hline 24 & 94.50 \\
\hline 25 & 43.50 \\
\hline 26 & 31.25 \\
\hline 27 & 26.50 \\
\hline 28 & 36.25 \\
\hline 29 & 50.00 \\
\hline 30 & 34.75 \\
\hline 31 & 48.25 \\
\hline 32 & 46.00 \\
\hline 33 & 36.75 \\
\hline 34 & 34.00 \\
\hline 35 & 26.00 \\
\hline 36 & 31.75 \\
\hline 37 & 29.75 \\
\hline 38 & 37.25 \\
\hline 39 & 26.75 \\
\hline 40 & 43.75 \\
\hline 41 & 27.25 \\
\hline 42 & 47.00 \\
\hline 43 & 41.00 \\
\hline 44 & 30.50 \\
\hline 45 & 45.25 \\
\hline 46 & 26.25 \\
\hline 47 & 45.50 \\
\hline 48 & 44.25 \\
\hline 49 & 48.75 \\
\hline 50 & 33.25 \\
\hline 51 & 27.50 \\
\hline
\end{tabular}

TABle 1: Continued.

\begin{tabular}{|c|c|}
\hline Position in ranking & Requirements (million dollars) \\
\hline 52 & 41.25 \\
\hline 53 & 29.50 \\
\hline 54 & 25.25 \\
\hline 55 & 40.00 \\
\hline 56 & 30.75 \\
\hline 57 & 39.00 \\
\hline 58 & 44.50 \\
\hline 59 & 47.50 \\
\hline 60 & 36.00 \\
\hline 61 & 28.50 \\
\hline 62 & 29.00 \\
\hline 63 & 30.25 \\
\hline 64 & 49.50 \\
\hline 65 & 33.00 \\
\hline 66 & 38.50 \\
\hline 67 & 33.50 \\
\hline 68 & 48.50 \\
\hline 69 & 35.00 \\
\hline 70 & 28.75 \\
\hline 71 & 25.50 \\
\hline 72 & 40.25 \\
\hline 73 & 38.75 \\
\hline 74 & 46.75 \\
\hline 75 & 37.00 \\
\hline 76 & 46.50 \\
\hline 77 & 44.00 \\
\hline 78 & 25.75 \\
\hline 79 & 38.25 \\
\hline 80 & 40.75 \\
\hline 81 & 42.75 \\
\hline 82 & 43.00 \\
\hline 83 & 32.25 \\
\hline 84 & 37.75 \\
\hline 85 & 44.75 \\
\hline 86 & 27.00 \\
\hline 87 & 39.50 \\
\hline 88 & 30.00 \\
\hline 89 & 37.50 \\
\hline 90 & 49.00 \\
\hline 91 & 41.75 \\
\hline 92 & 39.25 \\
\hline 93 & 34.50 \\
\hline 94 & 49.75 \\
\hline 95 & 48.00 \\
\hline 96 & 29.25 \\
\hline 97 & 47.75 \\
\hline 98 & 42.25 \\
\hline 99 & 46.25 \\
\hline 100 & 39.75 \\
\hline Total & 5542.00 \\
\hline
\end{tabular}


TABLE 2: Some Pareto solutions.

\begin{tabular}{lc}
\hline Solution & $\left(n_{P \mathrm{r}}, n_{a}, n_{s}, n_{w}\right)$ \\
\hline 63 & $(22,0,0,0)$ \\
91 & $(24,0,13,0)$ \\
93 & $(25,0,16,0)$ \\
100 & $(26,0,27,0)$ \\
110 & $(26,0,27,0)$ \\
112 & $(27,0,31,0)$ \\
191 & $(28,0,44,0)$ \\
114 & $(29,0,57,0)$ \\
252 & $(30,0,70,0)$ \\
48 & $(31,0,78,0)$ \\
117 & $(31,0,78,0)$ \\
107 & $(32,0,86,0)$ \\
286 & $(33,0,103,0)$ \\
198 & $(34,3,114,0)$ \\
25 & $(34,6,112,0)$ \\
123 & $(35,6,129,0)$ \\
400 & $(36,12,156,1)$ \\
154 & $(37,16,170,0)$ \\
155 & $(38,24,182,2)$ \\
30 & $(37,25,159,1)$ \\
\hline
\end{tabular}

TABLE 3: Different value systems expressed by criterion weights.

\begin{tabular}{lccccccccc}
\hline Coalition & $w_{1}$ & $w_{2}$ & $w_{3}$ & $w_{4}$ & $w_{5}$ & $w_{6}$ & $w_{7}$ & $w_{8}$ & $w_{9}$ \\
\hline $\mathrm{Co}_{1}$ & 0.23 & 0.14 & 0.11 & 0.14 & 0.11 & 0.07 & 0.09 & 0.07 & 0.04 \\
$\mathrm{Co}_{2}$ & 0.14 & 0.23 & 0.07 & 0.11 & 0.14 & 0.09 & 0.07 & 0.11 & 0.04 \\
$\mathrm{Co}_{3}$ & 0.11 & 0.09 & 0.14 & 0.11 & 0.23 & 0.14 & 0.07 & 0.04 & 0.07 \\
$\mathrm{Co}_{4}$ & 0.07 & 0.11 & 0.23 & 0.04 & 0.14 & 0.09 & 0.14 & 0.07 & 0.11 \\
$\mathrm{Co}_{5}$ & 0.04 & 0.07 & 0.23 & 0.11 & 0.14 & 0.09 & 0.14 & 0.07 & 0.11 \\
\hline
\end{tabular}

TABLE 4: Best portfolios from the five coalitions (in thousands).

\begin{tabular}{lccccccccc}
\hline Coalition & $N_{1}$ & $N_{2}$ & $N_{3}$ & $N_{4}$ & $N_{5}$ & $N_{6}$ & $N_{7}$ & $N_{8}$ & $N_{9}$ \\
\hline $\mathrm{Co}_{1}$ & 820 & 620 & 675 & 1095 & 1005 & 810 & 1086 & 660 & 630 \\
$\mathrm{Co}_{2}$ & 820 & 615 & 690 & 1140 & 1020 & 720 & 1050 & 630 & 690 \\
$\mathrm{Co}_{3}$ & 810 & 795 & 485 & 1095 & 1020 & 735 & 876 & 546 & 858 \\
$\mathrm{Co}_{4}$ & 820 & 670 & 585 & 1230 & 900 & 660 & 846 & 666 & 714 \\
$\mathrm{Co}_{5}$ & 750 & 780 & 585 & 1035 & 1005 & 795 & 924 & 672 & 756 \\
\hline
\end{tabular}

and falling within a frame of an approved general budget. Although the priorities of the citizenship have been identified and are likely to be reflected in a ranking of public policy actions, it is still necessary to solve a portfolio problem of public projects that should implement the agreed agenda.

Portfolio selection is an optimization problem with exponential complexity. The set of possible portfolios is the power set of the projects applying for funding. The cardinality of the set of portfolios is $2^{N}$, where $N$ is the number of projects. If synergetic projects and nonlinear and timing distributions effects are considered, the complexity of the resulting optimization model increases significantly. So far, the participatory budgeting exercises have been unable to deal with this kind of problem. Two questions arise. How to obtain the information about the preferences of the participants in the exercise? How to use it later to explore the space of portfolios and find the best solutions compatible with the participants' satisfaction?

Basic structures of collective decision making and information provided by participatory budgeting exercises are discussed in Section 2. The way in which a public portfolio can be optimized is described in Section 3. Section 4 details the way in which a portfolio can be optimized when the group preference information comes from a project ranking. Section 5 presents a portfolio optimization method when the preference information is given as individual goals. These methods are illustrated by real size examples in Sections 4 and 5. Finally, we draw some conclusions in Section 6.

\section{Some Background}

2.1. Basic Structures for Collective Decision Making. Let us consider a decision-maker group $(D M G)$ composed of $M$ actors with heterogeneous value systems. This collective is in charge of building a public project portfolio. According to Marakas [1], we can distinguish three basic structures for collective work oriented to decision making.

Figure 1(a) covers the case in which the whole group is responsible for the decision, there is a complete symmetry between the different $D M s$, and the final decision is made according to previously established rules that define the way in which the group is "constituted." Figure 1(b) corresponds to situations in which symmetry is lost, there exists a $D M$ in charge of the decision (hereinafter referred to as group supra-decision maker (GSDM)), who bases it on the opinions of the collective that processes a lower level of decision; the members of this collective only interact with the GSDM. The structure in Figure 1(c) shows a complete interaction amongst all participants, but the main responsibility still falls on the GSDM whose final judgment will be sustained by the best possible consensus of the members of the collective. All of the three structures assume a certain level of collaboration, even though it is not necessarily exempted from contradictions. These three cases will be referred to as partner association, team, and committee, respectively.

2.2. Information Stemming from the Participatory Exercise and Its Exploitation. Participatory budgeting exercises preferably take the form of Figure 1(c), with government representatives playing the role of GSDM. From this interaction, the GSDM can obtain the following preferential information from the actors representing the society:

(A) opinions about certain priorities that the public agenda should contain, accepted by a significant part of participants;

(B) individual preferences of each participant about the public policy actions requiring budgetary resources ordered in a ranking of priorities; 
TABLE 5: Best solutions $x_{G k}^{*}$ of problem (5) on the original criterion space (values in thousands).

\begin{tabular}{|c|c|c|c|c|c|c|c|c|c|}
\hline Portfolio & $N_{1}$ & $\mathrm{~N}_{2}$ & $N_{3}$ & $\mathrm{~N}_{4}$ & $N_{5}$ & $N_{6}$ & $N_{7}$ & $N_{8}$ & $N_{9}$ \\
\hline 1 & 820 & 685 & 690 & 1110 & 1005 & 810 & 936 & 666 & 750 \\
\hline 2 & 820 & 690 & 690 & 1095 & 1020 & 810 & 954 & 666 & 696 \\
\hline 3 & 820 & 685 & 690 & 1110 & 1020 & 810 & 936 & 678 & 720 \\
\hline 4 & 820 & 695 & 690 & 1095 & 1005 & 810 & 936 & 666 & 726 \\
\hline 5 & 820 & 670 & 695 & 1110 & 1005 & 810 & 936 & 672 & 720 \\
\hline 6 & 820 & 665 & 710 & 1110 & 1005 & 810 & 936 & 690 & 720 \\
\hline 7 & 820 & 680 & 690 & 1110 & 1020 & 810 & 954 & 666 & 720 \\
\hline 8 & 820 & 670 & 685 & 1110 & 1005 & 795 & 936 & 672 & 720 \\
\hline 9 & 820 & 685 & 695 & 1110 & 1005 & 810 & 936 & 690 & 726 \\
\hline 10 & 820 & 670 & 695 & 1095 & 1020 & 810 & 1002 & 666 & 672 \\
\hline 11 & 820 & 665 & 690 & 1125 & 1005 & 750 & 936 & 666 & 750 \\
\hline 12 & 820 & 670 & 690 & 1110 & 1005 & 810 & 936 & 672 & 720 \\
\hline 13 & 820 & 675 & 695 & 1095 & 1005 & 810 & 984 & 666 & 702 \\
\hline 14 & 820 & 675 & 690 & 1110 & 1005 & 810 & 936 & 666 & 696 \\
\hline
\end{tabular}

TABLE 6: Values of $\mu_{\text {sat }}\left(x_{G k}\right)$.

\begin{tabular}{lc}
\hline$x_{G k}$ & $\mu_{\mathrm{sat}}\left(x_{G k}\right)$ \\
\hline$x_{G 1}$ & 0.7967 \\
$x_{G 2}$ & 0.8510 \\
$x_{G 3}$ & 0.8693 \\
$x_{G 4}$ & 0.7818 \\
$x_{G 5}$ & 0.7818 \\
$x_{G 6}$ & 0.8693 \\
$x_{G 7}$ & 0.7818 \\
$x_{G 8}$ & 0.8415 \\
$x_{G 9}$ & 0.7691 \\
$x_{G 10}$ & 0.7654 \\
\hline
\end{tabular}

(C) if the public agenda success is measured through a set of indicators, the levels that each participant expects as a result of the budgeting exercise.

Given the social heterogeneity and conflicting interests, usually it is not possible to achieve consensuses in individual preferences (case B) or individual goals (C).

A portfolio is a subset of a set $P_{r}$ of projects or social actions which are under consideration. To distribute resources means to find a feasible portfolio that meets specific requirements. In practice, the GSDM is the one who solves the portfolio problem by using the obtained information. But the ethics of democracy (not only the electoral factor, but democracy as a government by and for the people) require the GSDM to adhere as much as possible to the preferential information stemming from the participatory exercises. It is noteworthy that in (A) and (B) social preferences are not collected over project portfolios, but over concrete actions. Since the budget distribution is a problem of project, policy, or public action portfolios, the issue is how to translate the information from participatory exercises into preferences over portfolios. In (C), the aspirations collected about the social status are likely to be contradictory among different participants, but social status indicators will vary as a result of the portfolio that is decided to be supported. Thus, the information in mode $(\mathrm{C})$ collects preferences over portfolios or over the result of portfolios.

Case $(\mathrm{A})$ is predominant among real exercises of participatory budgeting. The GSDM seeks to adapt his/her agenda previously conceived according to the opinions received and on that basis to make the distribution of budgetary resources. The mind of GSDM processes the information, partly modifies his/her own subjectivity, and decides the distribution of resources. The group representing the society is not the one solving the portfolio problem, but its opinions are taken into account.

In case (B), the public action rankings given by the participants are an expression of their preferences over projects, not over portfolios. Let us suppose that a method for integrating individual rankings is applied in collective ordering as the Borda score or a procedure based on exploitation of collective fuzzy preference relations (e.g., [2-4]). With the resulting group ordering, the GSDM has more information about social preferences regarding different actions that are to be budgeted. The GSDM shall use that information to find the best portfolio.

In the following section, we assume that the GSDM has at his/her disposal a ranking of priorities of public actions or projects stemming from the participatory exercise.

\section{Finding the Best Portfolio via Multiobjective Optimization}

As stated by Fernandez et al. [5] and Fernandez-Gonzalez et al. [6], the main difficulty for characterizing the "best public project portfolio" is finding a mechanism to appropriately define, evaluate, and compare social returns. Regardless 


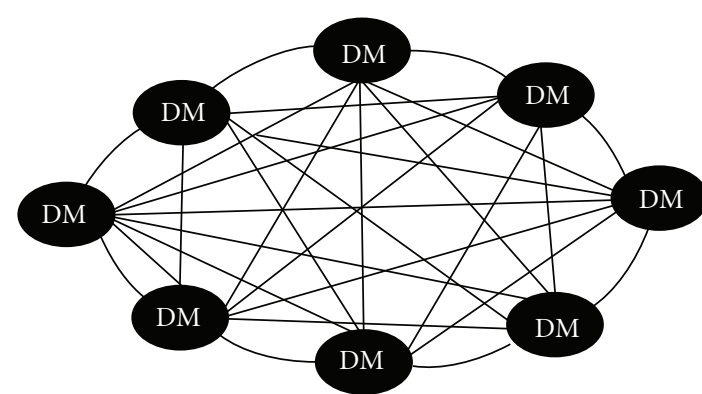

(a) Complete interaction and symmetry

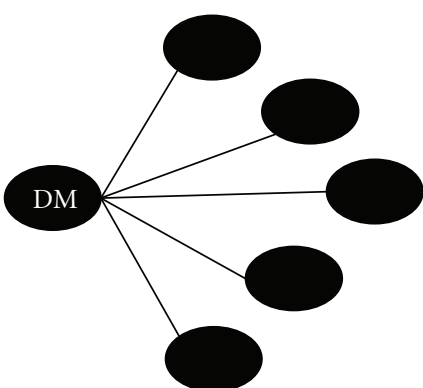

(b) A supra-decision maker interacting with the group

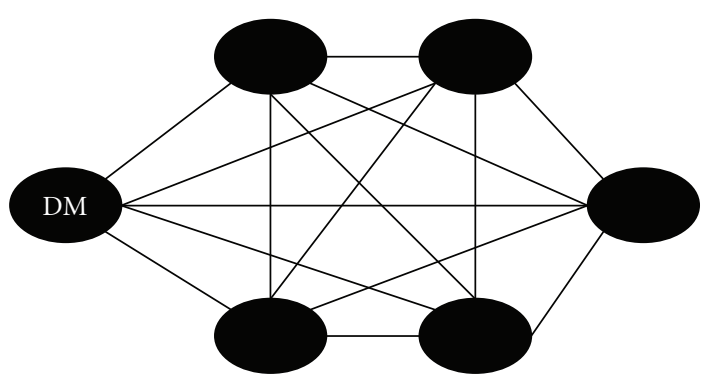

(c) Complete interaction, no symmetry

Figure 1: Basic structures for collective work.

of the varying definitions of the concept of social return, however, we can assert the tautological value of the following proposition.

Proposition 1. Given two social projects, $A$ and $B$, with similar costs and budgets, $A$ should be preferred to $B$ if $A$ has a better social return.

Ignoring, for a moment, the difficulties for defining the social return of a project portfolio, given two portfolios, $C$ and $D$, with equivalent budgets, $C$ should be preferred to $D$ if and only if $C$ has a better social return. Thus, the problem of searching for the best project-portfolio can be reduced to finding a method for assessing social project returns, or at least a comparative way to analyze alternative portfolio proposals. If we do not have reliable information about specific social project returns, the social impact of a given portfolio may be represented by a set of "proxy" variables measuring different criteria of portfolio quality. Let us denote by $\left\{v_{1}, \ldots, v_{K}\right\}$ such a set. Without loss of generality, we suppose that the GSDM's preferences are increasing with values of $v_{i}$.

Assumption 1. Let $C^{*}$ and $C^{* *}$ be two feasible portfolios and $V^{*}=\left(v_{1}, \ldots, v_{K}\right)^{*}$ and $V^{* *}=\left(v_{1}, \ldots, v_{K}\right)^{* *}$ their respective image in the proxy objective space. Then, $V^{*}$ is Paretodominated by $V^{* *}$ only if under the available information, $C^{* *}$ is preferred to $C^{*}$ from the GSDM's point of view.

Let $C$ be the set of feasible portfolios. Feasibility is determined by budgetary constraints. Assuming that the problem is solved by and for the GSDM, the comparison of the social value of the two portfolios becomes meaningful. Let
$C_{i}$ and $C_{j}$ be two feasible portfolios. Let us assume that $A_{P}$ is an asymmetric preference relation defined by the GSDM on a subset of $C \times C$. This is a subjective relation based on the information that the GSDM has about the portfolios. If $C_{i} A_{P} C_{j}$ does not generate a loop in $A_{P}$, then we can accept that $C_{i}$ has a greater value for the GSDM than $C_{j}$. The necessary condition for $C_{L}$ to be the best portfolio with respect to $A_{P}$ is that there is no $C_{i} \in C$ such that $C_{i} A_{p} C_{L}$. Furthermore, a sufficient condition for $C_{L}$ to be the best portfolio with respect to $A_{P}$ is $C_{L} A_{p} C_{j}$ for all $C_{j} \in C$.

Proposition 2. Under Assumption 1 the best portfolio for the GSDM is a nondominated solution to the problem

$$
\underset{C}{\operatorname{Maximize}}\left(v_{1}, \ldots, v_{K}\right) \text {. }
$$

The proof is very simple. Suppose that $C_{L}$ is the best portfolio being a dominated solution to problem (1). If $C_{L}$ is dominated by $C_{J}$, then from Assumption $1, C_{J} A_{p} C_{L}$. So, $C_{L}$ does not hold the necessary condition to be the best portfolio.

The choosing of a set $\left\{v_{1}, \ldots, v_{K}\right\}$ depends on the available information about the portfolio social impact, or how it is perceived by the GSDM. At least we should distinguish the two cases: (i) when the preference information from the participants has been aggregated in a ranking of priorities (case (B) of Section 2.2); (ii) when the public agenda success is measured by a set of indicators, and the contribution of each project or public action to the improvement of these indicators is known (case (C) of Section 2.2).

In the next section, we propose a way to build the set $\left\{v_{1}, \ldots, v_{K}\right\}$ and solve problem (1) in case (i). Case (ii) will be approached in Section 5. 


\section{Portfolio Selection When the Group Preference Information Comes from a Project (Action) Ranking}

It is necessary to compare the quality of the possible portfolios in order to find the best one. This problem was firstly approached by Fernandez et al. [5], under the assumption "the portfolio impact on a decision-maker's mind is determined by the number of supported projects and their particular rank." We shall accept that if project $a$ is clearly ranked better than $b$, then $a$ is admitted to have "more social impact" than $b$. The GSDM should take this information from the ranking into account. The appropriateness of a portfolio is not only defined by the quality of the included projects, but also by the amount of contained projects. As in Fernandez et al. [5], the purpose will be to find the best portfolio by increasing the number of supported projects and controlling the possible disagreements with respect to the GSDM's preferences, which as it is assumed, are incorporated in the input ranking. Some discrepancies may be acceptable between the information provided by the ranking and the decisions concerning the approval (hence, supporting) of projects, whenever this fact increases the number of projects in the portfolio. However, this inclusion should be controlled because the admission of unnecessary discrepancies is equivalent to underestimating the ranking information.

Among different heuristics, we suggest one that is based on dividing the ranking in five categories; then, on this basis construct the specific preference relation. The categories are labeled (1) vanguard, (2) high-medium, (3) medium, (4) lowmedium, and (5) rearguard. If $P_{r}$ is the set of projects, let us define a "priority" function $\rho: P_{r} \rightarrow\{1,2,3,4,5\}$ whose image is the project label (for instance, $\rho(a)=1 \Leftrightarrow a$ is ranked in the vanguard).

Through exercising such categorization, we may build up the preference relations according to the ranking, as explained below.

Absolute preference (denoted by $a \gg b): a \gg b \Leftrightarrow \rho(a)<$ $\rho(b)$ and $|\rho(a)-\rho(b)| \geq 2$.

Strict preference $(a>b): a>b \Leftrightarrow((\rho(a)<\rho(b)$ and $|\rho(a)-\rho(b)|=1)$ or $(\rho(a)=\rho(b)$ and $a$ is ranked better than b).

Weak preference $(a>\sim b): a>\sim b \Leftrightarrow a$ and $b$ are ranked equal, but $b$ needs more funds than $a$.

Strict outranking $(a S b): S=\gg \cup>U>\sim$

Note that $S$ is an asymmetric relation expressing preference for one of the projects. Hence, a well-formed portfolio should be compatible with the information contained in $S$. Let $C$ be a project portfolio that obtains support, with $a S b$ and $a \notin C$. In such a case, the inclusion of $b$ into $C$ would constitute a discrepancy with respect to the information contained in $S$. Thus, each portfolio may be associated a discrepancy set in the following way:

$$
D=\left\{(a, b) \in P_{r} \times P_{r} \text { such that } a S b, b \in C, a \notin C\right\} .
$$

$D$ can be partitioned in

(i) a set of absolute discrepancies $D_{a}=\left\{(a, b) \in P_{r} \times P_{r}\right.$ such that $a \gg b, b \in C, a \notin C\}$; (ii) a set of strict discrepancies $D_{s}=\left\{(a, b) \in P_{r} \times P_{r}\right.$ such that $a>b, b \in C, a \notin C\}$;

(iii) a set of weak discrepancies $D_{w}=\left\{(a, b) \in P_{r} \times P_{r}\right.$ such that $a>\sim b, b \in C, a \notin C\}$.

Let $n_{a}, n_{s}$, and $n_{w}$ denote the respective cardinality of the above sets. Considering also the number of supported projects in $C$ (denoted by $n_{P r}$ ), problem (1) can be transformed into

$$
\operatorname{Max}_{C} n_{P r}, \quad \operatorname{Min}\left(n_{a}, n_{s}, n_{w}\right)
$$

and according to Proposition 2, the best portfolio should be selected by the GSDM among its nondominated solutions. It is him/her who will finally select the adequate compromise among the number of supported projects and the quality given by their rank. Compromise solutions will depend on the extent that the GSDM relies on the information provided by the rank ordering and facts such as pressures exerted on him/her to include high-ranked projects or to increase the number of approved projects, as well as his/her aversion to costly projects.

4.1. An Illustrative Example. As illustration, let us consider the problem of distributing 2.5 billion dollars among 100 projects, all of them deserving individual financing. Information about these projects (costs in million dollars and their ranks according to some preference ordering) is shown in Table 1 (the data are fictitious).

Financing all projects would amount more than 5.5 billions, but the available funds are not enough to support them all. The traditional heuristics for solving this problem consider giving support by following the rank order until the available resources run out. If this method was used, the resources would reach up to project 22, implying a total expenditure of 2.468 billion dollars.

Let us suppose that the GSDM is not averse to making contradictory decisions with respect to the ranking information, thus being prepared to accept some discrepancies in order to increase the number of supported projects. Hence, he/she wants to evaluate nondominated solutions to problem (3). However, this problem exhibits exponential complexity, which complicates its solution through classical techniques of multiobjective optimization even for a medium-size instances [5]. Since it is difficult to model GSDM preferences over its objective functions, it seems preferable to apply a method for building the Pareto optimal set, and from this result, the GSDM can select the best "compromise." For this reason we choose a multiobjective evolutionary algorithm (MOEA). One advantage of MOEAs is that they handle efficiently exponential complexity of the search space. According to Fernandez et al. [5], several works report successful experiments applying MOEAs to large 0-1 multiobjective knapsack problems. Unlike classical multiobjective optimization techniques, MOEAs are not sensitive to mathematical properties of the objective functions or the shape of the feasible region; moreover, they can obtain many nondominated solutions in only one run [7]. Here, we apply the NSGA-II (nondominated sorting 
genetic Algorithm) developed by Deb et al. [8], which is the benchmark of evolutionary multiobjective optimization. The NSGA-II performance is fairly sensitive to the number of objective functions, but this is not a main drawback for solving problem (3). Its pseudocode is shown in Pseudocode 1 (adapted from [9]).

Binary encoding was used; a " 1 " in the individual $m$ th allele means that the $m$ th project belongs to this particular portfolio. One-point crossover and the standard mutation operator were implemented (cf. [10]). Binary tournament selection was performed as in [11].

The approach for handling constraints is based on the principle that any individual satisfying the constraints is always better than any individual that does not. If the feasible individuals in the population are classified into $k$ nondominated classes, then any unfeasible individual is relegated to class $k+1$. This implies that in binary tournaments any feasible individual will have priority over any nonfeasible individual.

The parameters of the evolutionary search were: crossover probability $=1$; mutation probability $=0.02$; population size $=100$ number of generations $=500$.

A representative sample of the Pareto front is shown in Table 2. Solution $63\left(n_{P r}, n_{a}, n_{s}, n_{w}\right)=(22,0,0,0)$ corresponds to allocating funds according to the ranking. If the GSDM is willing to accept some minor discrepancies, he/she can select the solutions $\left(n_{P r}, n_{a}, n_{s}, n_{w}\right)=(25,0,16,0)$ or $(24,0,13,0)$. Represented in the decision variable space, these solutions are shown in Figure 2.

\section{Selection of Public Project Portfolios When the Information about Preferences Is Given in terms of Goals}

When an individual decision maker gives his/her particular project ranking, he/she is expressing preferences about projects, not about portfolios. In this section we are interested in the case when each member of a participatory group is able to express his/her preferences through a set of aspiration levels which reflect the desired state of the concerned social object. Since those levels result from the aggregated action of projects, such information reflects preferences about portfolios.

The best portfolio for a heterogeneous participatory group should be the most acceptable agreement. Let us recall after Fernandez et al. [4] some ideas about collective agreement. When a heterogeneous group is making decisions, situations may arise that impede, even with the existence of the "constitution" rules, the determination of an acceptable agreement. These situations arise due to strong contradictions amongst important coalitions of its members, for example, when a solid opposition of a numerically significant minority exists and has to accept a preference that is supported by a weak majority of the group. To establish a collective agreement is only possible when unanimity or an appreciable level of consensus exists (cf. [4]). A definition of this last concept may be associated to the satisfying of the following two conditions. (a) There is an important agreeing majority.

(b) There is no appreciable disagreeing minority.

Fernandez et al. [4] alerted about the undesirable effects of the "majority dictatorship." This could have a negative influence on the stability of the group, making it less governable. Such "dictatorship" could be acceptable as an organizational principle with simple applications and that bases its rationality in the idea that more individuals should have weight more than that of lesser individuals (such a judgment ignores the importance of disagreement intensity). "Majority dictatorship" performs well in collectives that are disciplined and stable, mostly if the group is fundamentally comfortable accepting the majority principle as a basis for its decisions, and/or mechanisms for motivation and coercion exist in order to support group stability over the potential inconformity of a minority. As acceptable as the organizing principle might be, one should not make the mistake of assigning an ontological value that is generated by its rationality for every organization. Rejecting "majority dictatorship," the best agreement should maximize the group satisfaction and minimize the strong opposition. We need to define what a satisfactory/unsatisfactory portfolio is for each individual decision maker, followed by a way to find a good compromise between measures of satisfaction and unsatisfaction for the group.

In order to make a formal definition of satisfaction/dissatisfaction, let us introduce several new assumptions.

Assumption 2. Each individual decision maker in the participatory event is able to set a goal vector $\mathbf{x}^{*} \in \mathbb{R}^{K}$ which contains his/her aspiration levels for the relevant criteria of the concerned social object.

Assumption 3. Each individual decision-maker in the participatory event is able to set a set of constraints which express budgetary limits and some particular preferences concerning classes of projects, social groups, or geographic regions. Let us denote by $R_{F i}$ the corresponding feasible region in the portfolio space.

Assumption 4. Each member of the participatory group can set a fuzzy preference relation $\sigma_{i}(\mathbf{x}, \mathbf{y})$ defined on the criterion space; $\sigma_{i}$ values on $[0,1]$ represent the degree of credibility of the statement " $\mathbf{x}$ is at least as good as $\mathbf{y}$."

In order to be computable from the multicriteria description of $\mathbf{x}$ and $\mathbf{y}, \sigma_{i}(\mathbf{x}, \mathbf{y})$ can be built by using outranking methods [12], as is shown in Section 5.2.

Assumption 5. Each one of the group coalitions $\mathrm{Co}_{i}$ is a sufficiently homogeneous group as to achieve a consensus on the fuzzy preference relation $\sigma_{i}$ parameters, the constraint setting, the projects which are considered very important ones, and the goal vector $\mathbf{x}_{i}^{*}$.

Assumption 6. The GSDM can set a real value $\lambda(0.5<\lambda<1)$ such that given $(\mathbf{x}, \mathbf{y})$ in the criterion space if $\sigma_{i}(\mathbf{x}, \mathbf{y}) \geq \lambda$, the 


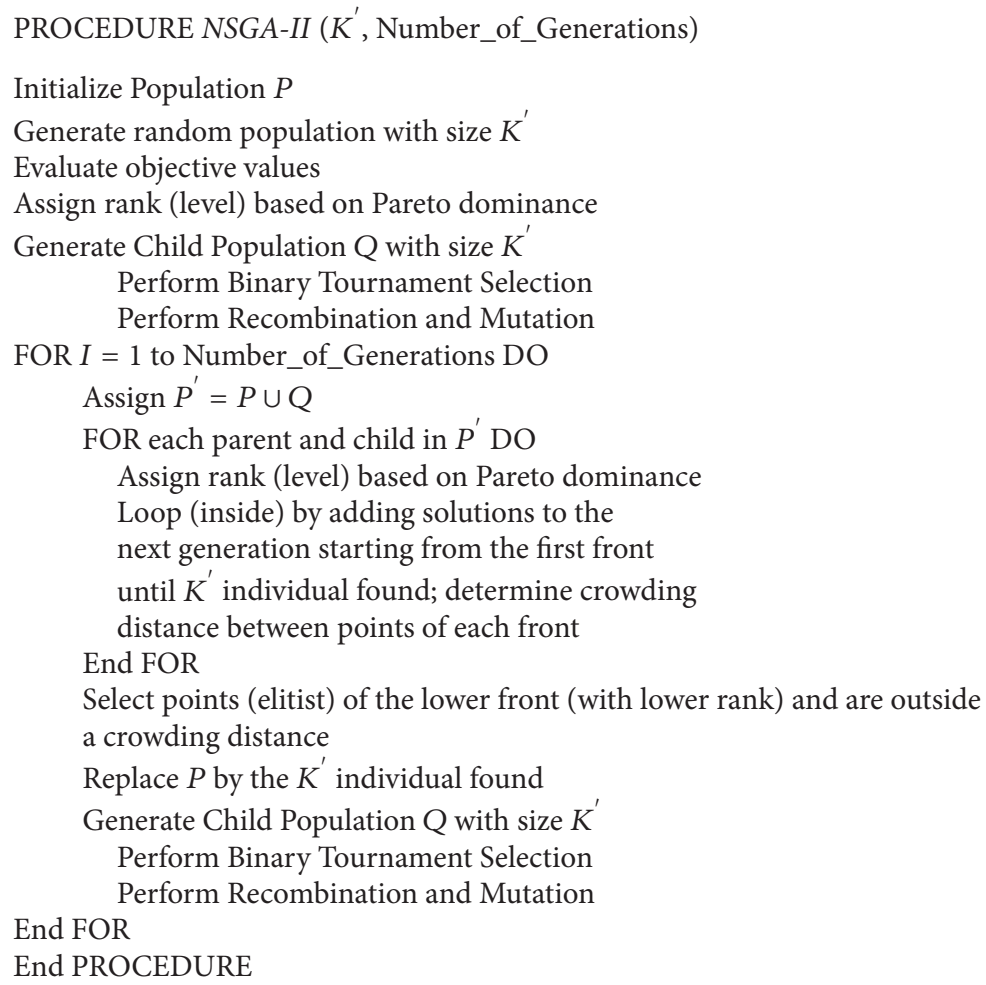

PseUdocode 1

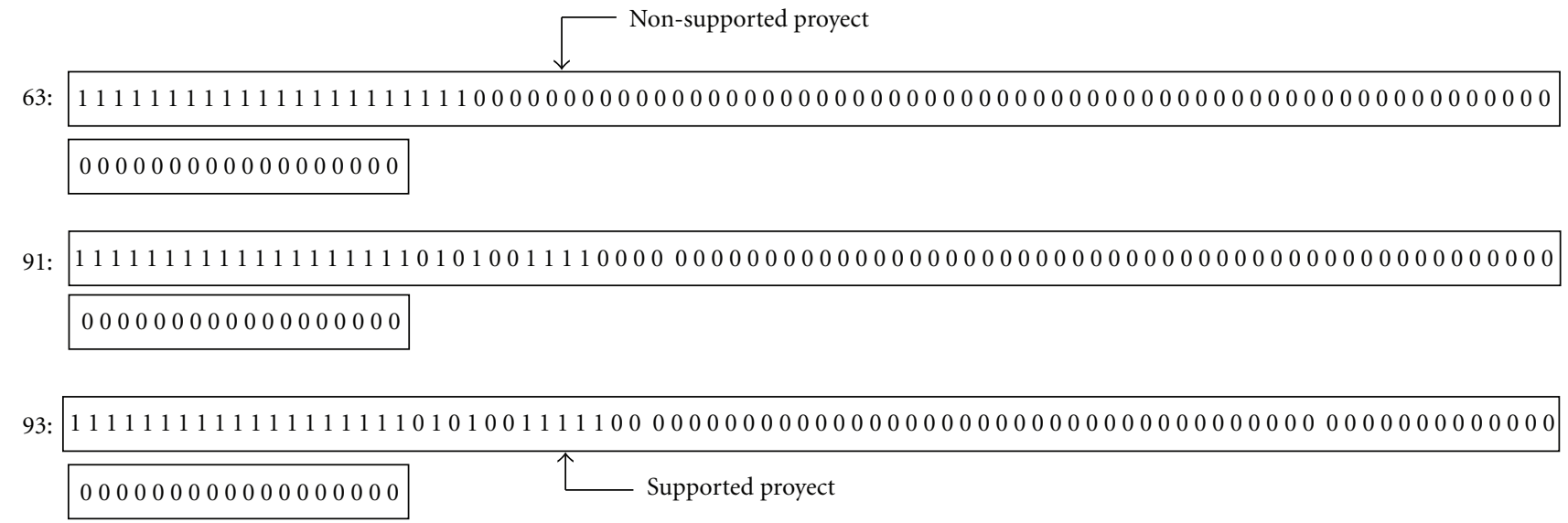

FIgURE 2: Some acceptable solutions in the decision space.

$i$ th decision maker (or the $i$ th coalition) may accept that " $\mathbf{x}$ is at least as good as y."

Definition 3. Let $\mathbf{x}_{i}^{*}$ be the goal vector set by the $i$ th decisionmaker (or the $i$ th coalition). Suppose that $C_{G}$ is a portfolio which is being considered by the group; $\mathbf{x}_{\mathbf{G}}$ is its image in the criterion space. The $i$ th decision maker (or the $i$ th coalition) is said to be satisfied by $\mathbf{x}_{\mathbf{G}}$ if and only if the following conditions hold:

(i) $\sigma_{i}\left(\mathbf{x}_{\mathbf{G}}, \mathbf{x}_{i}^{*}\right) \geq \lambda$; (ii) $\mathbf{x}_{\mathbf{G}}$ holds the constraints imposed by the $i$ th decisionmaker;

(iii) The projects which are considered very important by the $i$ th decision maker belong to $C_{G}$.

Definition 4. The ith decision-maker (or the ith coalition) is said to be unsatisfied by $\mathbf{x}_{\mathbf{G}}$ if one of the following conditions hold:

(i) $\sigma_{i}\left(\mathbf{x}_{\mathbf{G}}, \mathbf{x}_{i}^{*}\right)<0.5$ and $\sigma_{i}\left(\mathbf{x}_{i}^{*}, \mathbf{x}_{\mathbf{G}}\right) \geq \lambda$; 
(ii) $\mathbf{x}_{\mathbf{G}}$ does not hold the constraints imposed by the $i$ th decision maker;

(iii) most of the projects which are considered very important by the $i$ th decision maker do not belong to $C_{G}$;

Let $R_{F}$ be the union of the feasible regions $R_{F i}$. Note that two integer functions $n_{\text {sat }}\left(C_{G}\right)$ and $n_{\text {dis }}\left(C_{G}\right)$ may be defined on $R_{F}$. For each $C_{G}$ in $R_{F}$ with image $\mathbf{x}_{\mathbf{G}}$ in the criterion space, there is a number $n_{\text {sat }}$ obtained by counting the number of individual decision-makers which are satisfied by $\mathbf{x}_{\mathbf{G}}$. On the other hand, $n_{\text {dis }}$ is the result of counting the unsatisfied members. Note that $n_{\text {dis }}+n_{\text {sat }} \leq M$. The difference $M-$ $\left(n_{\text {dis }}+n_{\text {sat }}\right)$ is the number of decision makers which do not support a favorable consensus but are not against it.

The best agreement may be seen as the best compromise solution for the problem

$$
\begin{gathered}
\text { Maximize } n_{\text {sat }}, \quad \text { Minimize } n_{\mathrm{dis}} . \\
C_{G} \in R_{F} .
\end{gathered}
$$

Group satisfaction and dissatisfaction are optimized by solving problem (4). Dominated solutions of (4) should not be good agreements. Only nondominated solutions of (4) satisfying a minimum level of satisfaction should be considered. At least the condition of "agreeing relative majority" $n_{\text {sat }},>n_{\text {dis }}$ is necessary. So, (4) can be transformed into

$$
\begin{aligned}
& \text { Maximize } n_{\text {sat }}, \\
& \text { Minimize } n_{\text {dis }} \\
& \text { s.t. } \quad C_{G} \in R_{F} \\
& \\
& \quad n_{\text {sat }}>n_{\text {dis. }} .
\end{aligned}
$$

For solving (5), any non-linear multiobjective optimization method able to generate the Pareto frontier is acceptable. Once the set $D$ of nondominated solutions has been found, the GSDM should determine the "best" compromise. Such a most acceptable solution corresponds to the current stage of the group members' preferences and beliefs.

Unfeasibility of (5) should be a consequence of very conflicting value systems within the group. This is a tough situation which must be treated with care. The reasons for strong conflict should be determined. Further group discussions and the application of groupware techniques (e.g., $[13,14])$ would be necessary to close divergent beliefs, preferences, and constraint settings in order to achieve feasible solutions.

5.1. An Evolutionary Algorithm to Solve Problem (5). Solving problem (5) is not an easy task due to its size and the nonlinearity of its objective functions. We use evolutionary multiobjective optimization. Evolutionary algorithms are less sensitive to some mathematical properties of objective functions and constraints than the traditional optimization methods [9]. Additionally, in problem (5) an evolutionary algorithm helps to handle its complexity with respect to the number of applicant projects because computer efficiency of

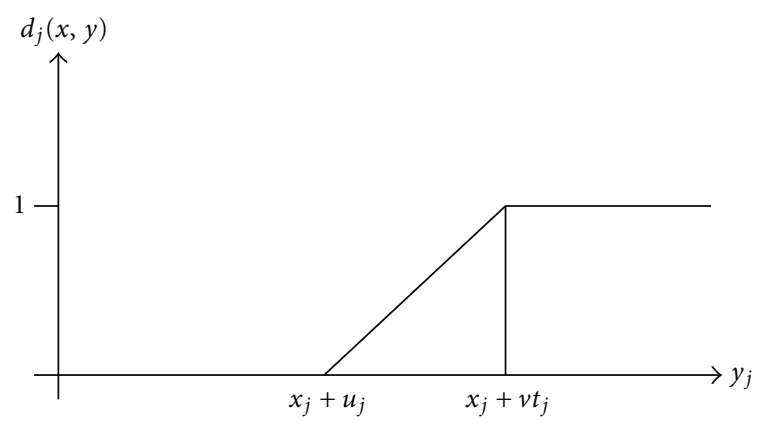

FIgURE 3: Partial discordance relation $\mathbf{d}_{\mathbf{j}}(x, y)$.

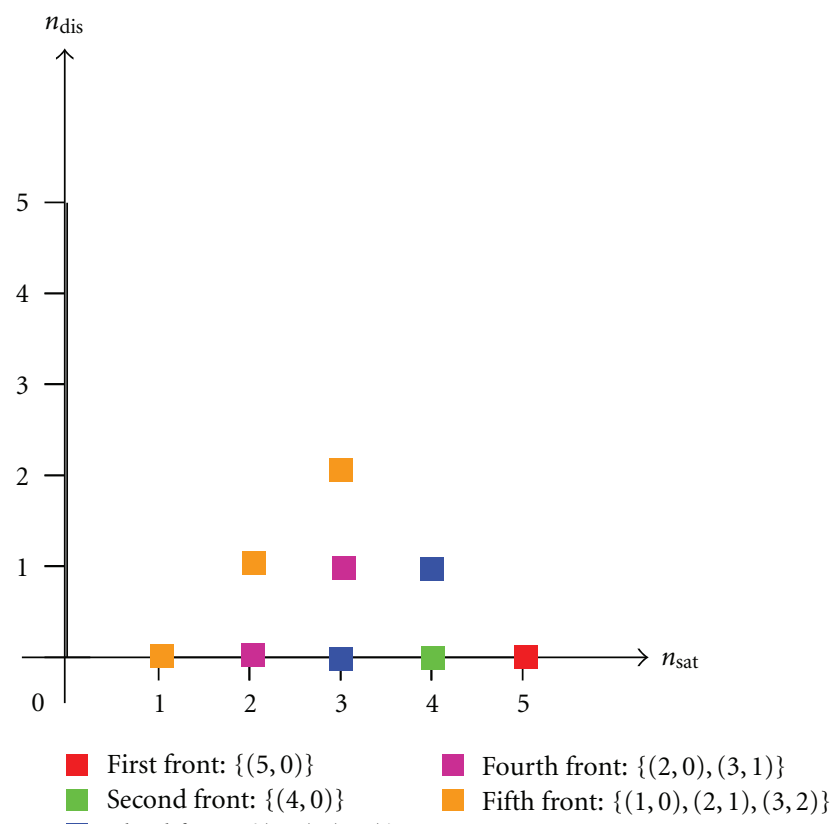

FigurE 4: Several NSGA-II's nondominated fronts of problem (5).

these methods is less sensitive to problem size in comparison to the traditional techniques.

Algorithm 1 is a direct adaptation of the NSGA-II [11].

In the binary tournament, two individuals are chosen randomly, the winner being the one with a smaller "rank." If both are in the same front, the tournament is won by the one who has a bigger crowding distance.

5.2. Example: Resources Assignment for Social Action Programs (Projects). This example is a fictitious decision making situation in which the participatory group chooses among 100 different social policies (projects), each with a direct social return. This is measured by using a nine-component vector $\left(N_{1}, N_{2}, \ldots, N_{9}\right) . N_{i}=n_{k j}$, the number of people belonging to the $j$ th social category which receive the $k$ th benefit level from that policy or project. In this example $j=$ $1,2,3$ correspond to (extreme poverty, poverty, low-middle), and $k=1,2,3$ to (high impact, middle impact, low impact). 


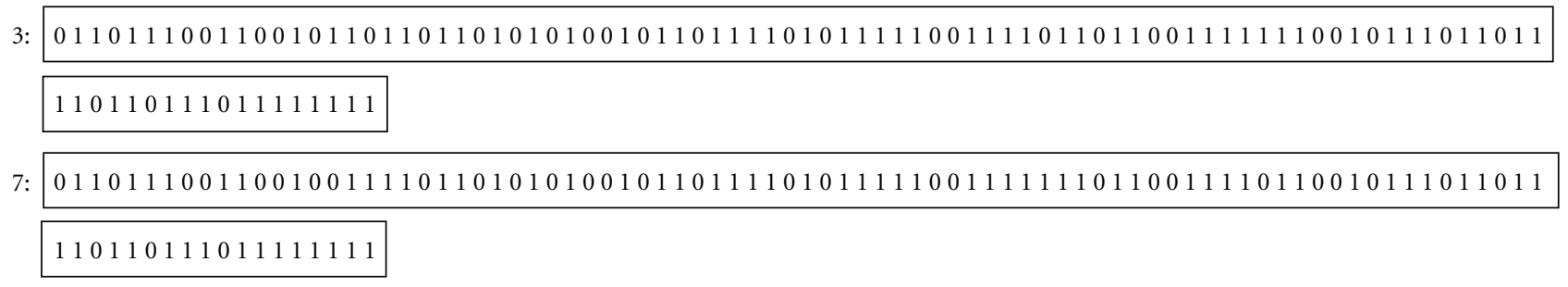

FIGURE 5: Best solutions in the decision variable space.

$N_{1}, N_{2}$, and $N_{3}$ correspond to extreme poverty people; $N_{7}$, $N_{8}$, and $N_{9}$ concern low-middle class.

$N_{i}^{m}$ denotes the value of $N_{i}$ associated to the $m$ th project. Let $C$ be a portfolio (a subset of projects which receives financial support). The value of $N_{i}$ for the whole portfolio is calculated as $N_{i}(C)=z_{1} N_{i}^{1}+\cdots+z_{100} N_{i}^{100}$, where $z_{m}=1$ if the $m$ th project is supported, and $z_{m}=0$ otherwise. The aim of this decision problem is to choose the "best" portfolio satisfying budget constraints. These are imposed by the type of project (educational, health, etc.), geographic region, and the whole portfolio. The total available budget was set as Total_budget $=2.5$ billion dollars. The constraints by class and region are given by

0.3 Total_budget $\leq$ Budget_Class $1 \leq 0.4$ Total_budget,

0.25 Total_budget $\leq$ Budget_Class $2 \leq 0.35$ Total_budget,

0.2 Total_budget $\leq$ Budget_Class $3 \leq 0.3$ Total_budget,

0.4 Total_budget $\leq$ Budget_Region $1 \leq 0.6$ Total_budget,

0.4 Total_budget $\leq$ Budget_Region $2 \leq 0.6$ Total_budget.

The degree of truth $\sigma(\mathbf{x}, \mathbf{y})$ of the statement " $\mathbf{x}$ is at least as good as $\mathbf{y}$ " was calculated as in the ELECTRE-III method (cf. [12]); that is,

$$
\sigma(\mathbf{x}, \mathbf{y})=c(\mathbf{x}, \mathbf{y}) N(d(\mathbf{x}, \mathbf{y}))
$$

where $c(\mathbf{x}, \mathbf{y})$ denotes the degree of truth of the concordance predicate given by $(7)$ and $N(d(\mathbf{x}, \mathbf{y}))$ denotes the degree of truth of the nondiscordance predicate

$$
c(\mathbf{x}, \mathbf{y})=\sum_{j \in C_{x, y}} w_{j},
$$

where $C_{x, y}$ is the concordance coalition and $w$ 's denote "weights" $\left(w_{1}+w_{2}+\cdots+w_{n}=1\right)$.

Let $D_{x, y}$ be the discordance coalition with " $\mathbf{x}$ is at least as good as y." The intensity of discordance is measured in comparison with a veto threshold $v t_{j}$, which is the maximum difference $y_{j}-x_{j}$ compatible with $\sigma(\mathbf{x}, \mathbf{y})>0$. The model for the nondiscordance predicate is based on (8) (cf. [15]) shown below:

$$
\begin{gathered}
N(d(\mathbf{x}, \mathbf{y}))=\min _{j \in D_{x, y}}\left[I-d_{j}(\mathbf{x}, \mathbf{y})\right], \\
d_{j}(x, y)= \begin{cases}1 & \Longleftrightarrow \nabla_{j} \geq v t_{j}, \\
\frac{\left(\nabla_{j}-u_{j}\right)}{\left(v t_{j}-u_{j}\right)} & \Longleftrightarrow u_{j}<\nabla_{j}<v t_{j}, \\
0 & \Longleftrightarrow \nabla_{j} \leq u_{j},\end{cases}
\end{gathered}
$$

where $\nabla_{j}=y_{j}-x_{j}$ and $u_{j}$ is a discordance threshold (see Figure 3).

Let us admit that the participatory group is composed of five coalitions with conflicting value systems. Coalitions $\mathrm{Co}_{1}, \mathrm{Co}_{2}, \ldots, \mathrm{Co}_{5}$ are composed by $10,9,8,7,6$ members, respectively $(M=40)$. Each value system is characterized by a different vector of weights as shown in Table 3. According to Assumption 5, a particular best portfolio was obtained for each value system. Those solutions are shown in Table 4.

In order to apply Condition (iii) in Definition 3, we proceeded as follows. For each coalition, the set of projects in its particular best portfolio was ranked according to their contribution to the most important criterion (for instance, Criterion 1 for $\mathrm{Co}_{1}$, Criterion 2 for $\mathrm{Co}_{2}$, Criterion 5 for $\mathrm{Co}_{3}$, etc.). After that, the best ranked 10 projects were chosen as "very important projects" for the corresponding coalition. $\lambda$ was set to 0.67 . The models of $\sigma_{j}$ were built by using the weights given in Table 3; the indifference thresholds were calculated as a measure of the error evaluating each objective, which was assessed as the $15 \%$ of its maximum value on the set of projects; the veto threshold for the $i$ th criterion was settled as $0.5^{*}\left(\operatorname{Max} N_{i}-\operatorname{Min} N_{i}\right)$; operations Max and Min act on a population.

We used the evolutionary algorithm of Section 5.2 for solving problem (5). Binary encoding was chosen; a " 1 " in the individual $m$ th allele means that the $m$ th project belongs to this particular portfolio. Other parameters of the evolutionary search were crossover probability $=1$; mutation probability $=0.02$; population size $=100$.

Several nondominated fronts of the last NSGA-II's population in the objective space of problem (5) are shown in Figure 4 . In this space, a single nondominated solution $n_{\text {sat }}=$ 5 and $n_{\text {dis }}=0$ was obtained; that is, the five coalitions are considered satisfied. After 20 runs, 500 generations per run, 14 nondominated points in the portfolio space were detected, which correspond to the solution $(5,0)$. Their values in the 
original nine-dimensional criterion space are pointed out in Table 5.

Any of these portfolios is unanimously satisfactory for the group, but only one can be selected. When there are several solutions of problem (5) in the portfolio space, the GSDM should apply a voting rule or additional criteria for choosing a single one.

Taking into account the indifference thresholds, solutions in rows fifth, eighth, twelfth, and fourteenth are strictly outranked by $\mathbf{x}_{G 1}^{*}$, so they should not be chosen as the best ones. Thus, the set shown in Table 5 is reduced to 10 solutions. On this set we propose to evaluate the fuzzy predicate "for all $j$, the $j$ th group member considers that $\mathbf{x}_{G k}{ }^{*}$ is at least as good as his/her particular goal vector $\mathbf{x}_{j}{ }^{*}$." It is the conjunction of the fuzzy predicates "the $j$ th group member considers that $\mathbf{x}_{G k}{ }^{*}$ is at least as good as his/her particular goal vector $\mathbf{x}_{j}{ }^{*}$." In [16] some desirable properties of the geometric mean as conjunction operator in the framework of the socalled "compensatory fuzzy logic" are discussed. Using the geometric mean for conjunction, the degree of truth of the first predicate can be calculated as

$$
\mu_{\text {sat }}\left(\mathbf{x}_{G K}\right)=\left(\prod_{j=1,2, \ldots, M} \sigma_{j}\left(\mathbf{x}_{G K} \mathbf{x}_{j}^{*}\right)\right)^{1 / M},
$$

where $\sigma_{j}$ denotes the fuzzy outranking function of the $j$ th member and $M$ is set to 40 . The values of $\mu_{\text {sat }}\left(\mathbf{x}_{G k}\right)$ are provided by Table 6 .

Solutions with greater values of $\mu_{\text {sat }}$ should be more satisfactory. However, $\mu_{\text {sat }}\left(z_{1}\right)>\mu_{\text {sat }}\left(z_{2}\right)$ may be not a sufficient condition to affirm that group satisfaction is greater with $z_{1}$. This can be approached by the concept of the fuzzy strict order, which can be used in order to compare truth values of statements modeled through predicates. A fuzzy strict order is a predicate $o: U^{2} \rightarrow[0,1]$ ( $U$ denotes the universe) that meets both of the following conditions $[16,17]$.

(A1) $o(x, y)=n[o(y, x)]$ (generalized fuzzy reciprocity) ( $n$ denotes the negation operator).

(B1) If $o(x, y) \geq 0.5$ and $o(y, z) \geq 0.5$, theno $(x, z) \geq$ $\max (o(x, y), o(y, z))(\max -\max$ fuzzy transitivity or strong stochastic transitivity).

With the definition provided by the above $(A 1)$ and $(B 1)$, the function

$$
o(x, y)=0.5[C(x)-C(y)]+0.5
$$

with $n(x)=1-x$ is a strict order over the universe of the predicate $C$ (cf. [18]), which has been already used successfully (cf. [17]). The predicate $o\left(\mu_{\text {sat }}\left(z_{1}\right), \mu_{\text {sat }}\left(z_{2}\right)\right)$ allows measuring "how more satisfactory is $z_{1}$ than $z_{2}$ for the group." $o\left(\mu_{\text {sat }}\left(z_{1}\right)\right.$, $\left.\mu_{\text {sat }}\left(z_{2}\right)\right)$ higher than 0.5 indicates a preference favoring $z_{1}$. If $o\left(\mu_{\text {sat }}\left(z_{1}\right), \mu_{\text {sat }}\left(z_{2}\right)\right) \approx 0.5$, indifference can be accepted.

Solutions $\mathbf{x}_{G 3}$ and $\mathbf{x}_{G 7}$ maximize $\mu_{\text {sat }}$. Using (10) we obtain $o\left(\mathbf{x}_{G 3}, \mathbf{x}_{G 7}\right)=0.50$ and $o\left(\mathbf{x}_{G 3}, \mathbf{x}_{G 2}\right) \approx 0.51$,
$o\left(\mathbf{x}_{G 2}, \mathbf{x}_{G 3}\right) \approx 0.49 . \mathbf{x}_{G 3}$ or $\mathbf{x}_{G 7}$ can be chosen as the best solution by the GSDM. Figure 5 shows these solutions in the decision variable space.

\section{Concluding Remarks}

Participatory budgeting construction exercises can be seen as a work of a committee, in which the executive plays the role of the group supra-decision-maker and the participants are representatives (formal or not) of the society. There are three main ways how the GSDM can collect information from the participatory group:

(A) as opinions about certain priorities that the public agenda should contain;

(B) as individual preferences of each participant about the public policy actions requiring budgetary resources, ordered in a ranking of priorities;

(C) as levels of individual aspirations with respect to the indicators that reflect the status of the social object.

The ethics of democracy requires from the GSDM to adhere as much as possible to the preferential information stemming from the participatory exercises. Since the budget distribution is a problem of the public project (actions) portfolio, the mode (A) offers very little preferential information, and the GSDM thus preserves huge freedom for his/her decisions. The mode (B) offers more preferential information; here the GSDM still has the solution of the portfolio problem on his/her hands, but the democracy ethics force him/her to seek solutions that are compatible with the ranking of priorities that stems from the participatory exercise.

There are two main situations in which a GSDM would find revenues in this approach: (1) he/she is averse to projects whose costs exceed the average considerably; and (2) he/she is strongly interested in increasing the number of supported projects. The procedure presented in Section 4 allows searching for reasonable compromises between the group priorities and the GSDM's interest in increasing the amount of public agenda actions.

The preferential information obtained from mode $(C)$ is sufficient to allow solving a portfolio problem by thoroughly exploring the space of solutions in order to maximize the satisfaction of the social representation. To the best of our knowledge, no other approach has been proposed for maximizing group satisfaction and minimizing dissatisfaction in portfolio optimization. The method described in Section 5 transforms a group multiobjective portfolio optimization problem into a group choice problem on a decision set composed of a relatively small set of alternative portfolios. This set contains the possible acceptable consensuses in the portfolio space. Once such a set has been identified, other well-known techniques can be used to reach the final solution. The main advantages are that (a) the search for the best agreement is not limited to portions of the Pareto frontiers and (b) no voting rule is used by the optimization algorithm; no, to some extent, arbitrary way of handling collective preferences is needed. Very good performance of the whole proposal is illustrated by a real-size example. 
Generate a size $n$ random population $P$, calculating the portfolio criteria.

Determine the different fronts assigning each individual a "rank" that is, the front which it belongs to and its crowding distance.

Execute the following as many times as generations

Generate an offspring population and calculate their criteria

Select the parents using binary tournament. Their crosses produce two descendants to whom the mutation operator is applied

Combine the parents' population and offspring population:

Evaluate the $\sigma$ value in GDM $\times P$. GDM is the group of $M$ decision-makers

Calculate the satisfaction and dissatisfaction level for each individual:

If

$\sigma_{i}\left(x_{G}, x_{i}^{*}\right) \geq \lambda_{i}$ and $x_{G}$ satisfies the $i$-DM restrictions; and all projects which $i$-DM considers very important belong to the portfolio,

Then

If

The individual $i$ is satisfied

$\sigma_{i}\left(x_{G}, x_{i}^{*}\right)<0.5$ and $\sigma_{i}\left(x_{i}^{*}, x_{G}\right) \geq \lambda$, or $a_{G}$ doesn't satisfy the $i$-DM restrictions or a Then significant part of projects that $i$-DM considers very important is not in $C_{G}$,

Count $n_{\text {sat }}$ and $n_{\text {dis }}$

The individual $i$ is unsatisfied

Determine the different fronts

Select the new population so that the members of the first fronts belong to it, and if necessary, execute the Crowding-Sort

Repeat the above-mentioned as another generation.

Algorithm 1

The role of GSDM becomes rather that of a group-work facilitator. If the collective is formed as a representative sample of the social structure, the solutions obtained by our method reflect the social preference, and the procedure may become a paradigm of the best forms of democracy.

\section{Acknowledgments}

The authors acknowledge the support from the Autonomous University of Sinaloa and the CONACyT. They express their gratitude for the constructive comments from two anonymous reviewers.

\section{References}

[1] G. M. Marakas, Decision Support Systems in the 21th Century, Prentice Hall, Upper Saddle River, NJ, USA, 1999.

[2] C. Macharis, J. P. Brans, and B. Mareschal, "The GDSS PROMETHEE Procedure. A PROMETHEE-GAIA based procedure for group decision support," Journal of Decision Systems, vol. 7, pp. 283-307, 1998.

[3] J. C. Leyva-López and E. Fernández-González, "A new method for group decision support based on ELECTRE III methodology," European Journal of Operational Research, vol. 148, no. 1, pp. 14-27, 2003.

[4] E. Fernandez, S. Bernal, J. Navarro, and R. Olmedo, "An outranking-based fuzzy logic model for collaborative group preferences," TOP, vol. 18, no. 2, pp. 444-464, 2011.

[5] E. Fernandez, L. F. Felix, and G. Mazcorro, "Multi-objective optimisation of an outranking model for public resources allocation on competing projects," International Journal of Operational Research, vol. 5, no. 2, pp. 190-210, 2009.

[6] E. Fernandez-Gonzalez, I. Vega-Lopez, and J. Navarro-Castillo, "Public portfolio selection combining genetic algorithms and mathematical decision analysis," in Bio-Inspired Computational Algorithms and Their Applications, S. Gao, Ed., pp. 139-160, INTECH, 2012.

[7] C. Coello, D. Van Veldhuizen, and G. Lamont, Evolutionary Algorithms for Solving Multi-Objective Problems, Kluwer Academic Publishers, New York, NY, USA, 2002.

[8] K. Deb, A. Pratap, S. Agarwal, and T. Meyarivan, "A fast and elitist multiobjective genetic algorithm: NSGA-II," IEEE Transactions on Evolutionary Computation, vol. 6, no. 2, pp. 182-197, 2002.

[9] C. Coello, D. Van Veldhuizen, and G. Lamont, Evolutionary Algorithms for Solving Multi-Objective Problems, Springer, New York, NY, USA, 2007.

[10] Z. Michalewicz, Genetic Algorithms + Data Structures=Evolution Programs, Springer, Berlin, Germany, 1996.

[11] K. Deb, Multi-Objective Optimization using Evolutionary Algorithms, John Wiley \& Sons, Chichester, UK, 2001.

[12] B. Roy, "The outranking approach and the foundations of ELECTRE methods," in Reading in Multiple Criteria Decision Aid, C. A. Bana e Costa, Ed., pp. 155-183, Springer, Berlin, Germany, 1990.

[13] P. Eklund, A. Rusinowska, and H. De Swart, "Consensus reaching in committees," European Journal of Operational Research, vol. 130, pp. 414-429, 2007. 
[14] J. L. Soubie and P. Zaraté, "Distributed decision making: a proposal of support through cooperative systems," Group Decision and Negotiation, vol. 14, no. 2, pp. 147-158, 2005.

[15] V. Mousseau and L. Dias, "Valued outranking relations in ELECTRE providing manageable disaggregation procedures," European Journal of Operational Research, vol. 156, no. 2, pp. 467-482, 2004.

[16] R. Espin, E. Fernandez, G. Mazcorro, J. Marx-Gomez, and M. I. Lecich, "Compensatory Logic: a fuzzy normative model for decision making," Investigación Operativa, vol. 27, no. 2, pp. 188-197, 2006.

[17] R. Espin, E. Fernandez, G. Mazcorro, and M. I. Lecich, "A fuzzy approach to cooperative n-person games," European Journal of Operational Research, vol. 176, no. 3, pp. 1735-1751, 2007.

[18] D. Dubois and H. Prade, Fuzzy Sets and Systems: Theory and Applications, Academic Press, 1980. 

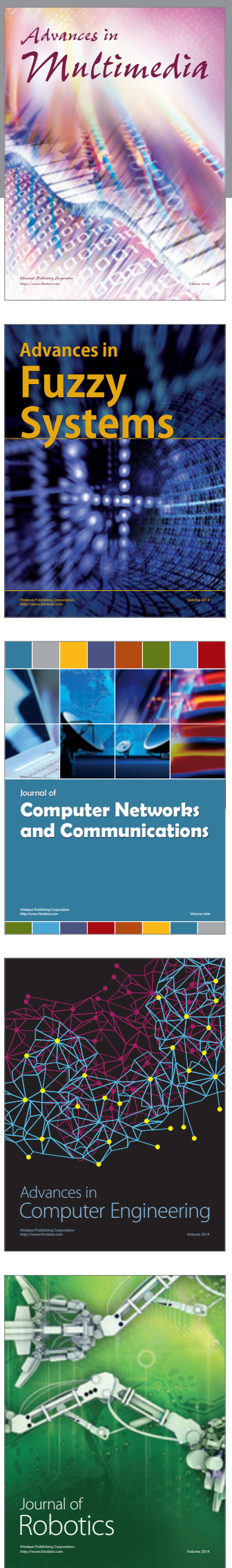

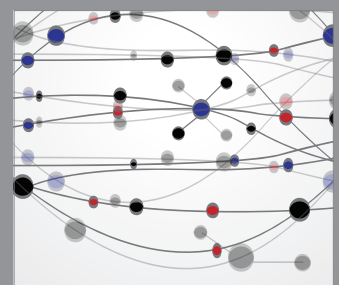

The Scientific World Journal
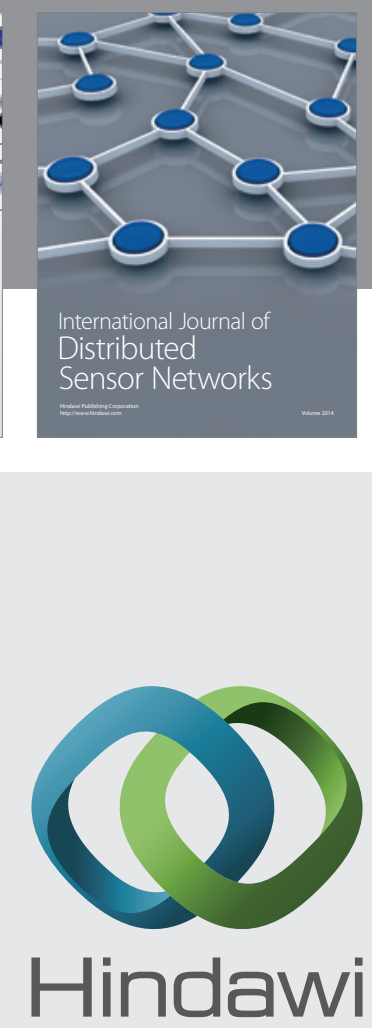

Submit your manuscripts at

http://www.hindawi.com
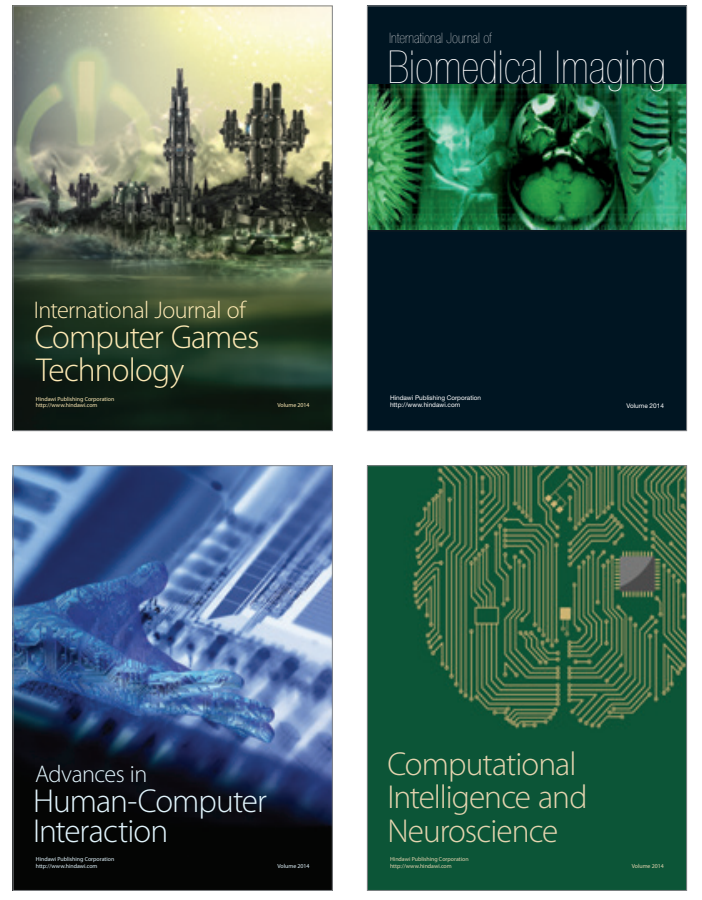
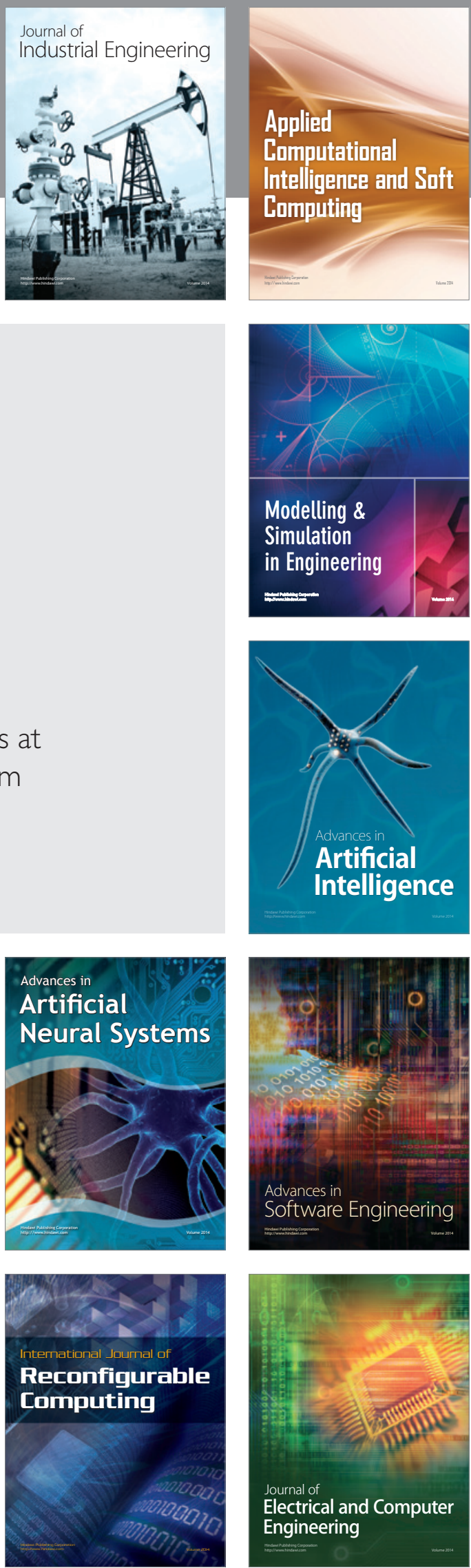